\title{
Should health technology assessment be more patient centric? If so, how?
}

\author{
Michael Drummond $^{1,2} \cdot$ Aleksandra Torbica $^{2} \cdot$ Rosanna Tarricone $^{2}$
}

Published online: 16 April 2020

(c) Springer-Verlag GmbH Germany, part of Springer Nature 2020

\section{Introduction}

Health technology assessment (HTA) methods and processes have been criticized for not being sufficiently 'patient centric'. For example, Perfetto [1] argued that a proposed approach for assessing the value of health care interventions had not sufficiently incorporated a patient perspective and suggested that it represented a 'missed opportunity'. A similar point was made about the other 'value assessment frameworks' developed recently in the United States [2]. In addition, Slejko et al. [3] proposed some key elements of a 'patient informed' reference case for conducting economic evaluations, which would supplement reference cases outlined by groups such as the second Panel on Cost-Effectiveness in Health and Medicine [4], by including consideration of elements such as convenience in receiving care, effects on the patient's family, examination of whether quality of life instruments include the most relevant domains and a model structure for the economic evaluation that adequately reflects the patient's journey through the various treatment options.

While the case to consider the patient perspective is strong, the way in which it should be incorporated in HTA is not obvious. Most HTA analysts would argue that HTAs, with the possible exception of some that are undertaken to support the development of clinical guidelines, or initiatives such as shared (clinical) decision-making, are conducted for those making decisions about the allocation of health care resources for a given population. This population may be the enrollees of a given health plan or, in the case of national health services or national insurance schemes, the whole population of a given country. The population would include patients who currently have the disease of interest,

Michael Drummond

mike.drummond@york.ac.uk

Centre for Health Economics, University of York, York, UK

2 SDA Bocconi School of Management, Bocconi University, Milan, Italy their families, those who have been patients in the past and those who may contract the disease in the future, as well as those past, present and future sufferers of other diseases.

Of course, there may be considerable similarity between the perspective of patients currently suffering from a disease and the population at large, but this is not necessarily the case $[5,6,7]$. Therefore, making HTA more patient centric may not be as simple as it appears. The case for considering the patient perspective may differ by type of health care system [8]. For example, in a private insurance-based system, where a substantial proportion of the payments may be made directly by the patients, one might expect more consideration of the patient perspective than in a publicly funded national health service.

The empirical evidence generated in the recent years shows that there is greater awareness about the importance of patients' views in HTA, but there is no common understanding of what "patient-centric HTA" actually means [9]. Here, we attempt to shed further light on the issue by conceptualizing "patient centricity" in two ways: (a) encouraging patients' engagement in HTA process and (b) enlarging the scope of evidence in HTA to include patients' outcomes and preferences (HTA methods). We discuss the opportunities and challenges of each, by providing some recent examples from different countries. Finally, we discuss some additional ways to make HTA more patient centric.

\section{Encouraging patient participation in HTA processes}

Many HTA organizations have made attempts to involve patients in their processes. This participation has taken many forms, including membership of committees, commenting on draft reports, inclusion in scoping exercises (to plan particular assessments) and citizens' forums (to advise on particular ethical issues) $[10,11]$. The main concern of 
patient organizations is that these initiatives are not as effective as they could be.

First, the involvement of patients can best be described as 'reactive' rather than 'proactive', in that by the time comments are requested on the results of particular HTAs it is often too late to re-visit the design of the study or the data collection. It is therefore important to involve patient organizations at an earlier stage, when it might be possible to influence the scope or methodology of the study [12].

Secondly, the resources required to produce comments in a timely fashion, or to participate effectively on committees, are often beyond the reach of many patient organizations. Therefore, the influence of the patient perspective in HTA activities may be less that that of other stakeholders, such as the clinical professions or the healthcare industry. However, attempts have been made to increase the effectiveness of patient participation, such as the European Patients Academy (https://www.eupati.eu), where patient organizations can gain access to training on the key elements of HTA.

\section{Enlarging the scope of evidence in HTA}

\section{Incorporating patient-reported measures in the development of 'value frameworks'}

There are currently relatively few examples of this, since in many jurisdictions there is a firm belief that population preferences, either direct or exercised through public decisionmaking, should be the main driver of HTA. However, there may be opportunities for more patient involvement without infringing this more general principle. For example, in their argument for a 'Patient-Informed Reference Case', Slejko et al. [3] argue that a societal perspective for economic evaluation, which is already adopted in some jurisdictions (e.g. Sweden), allows the consideration of patients' costs. In addition, the ISPOR Value Assessment Framework for the US [13], while arguing the starting point should be an estimate of incremental cost per QALY, suggested that there should be more investigation of the measurement and incorporation of 'novel' elements of value.

Many of these elements of value, such as impacts on work participation, increased convenience in receiving care, insurance value, the value of hope and impacts on caregivers, would be of direct interest to patients [14]. Other authors argue that value "lies in the eye of the patient" [15], suggesting that patient-reported outcome measures (PROMs) are an invaluable source of data that can and should be used to inform HTA. The use of PROMs to support label claims for regulatory decision has been endorsed by FDA in the US, but there is still limited evidence of their use by HTA bodies. The same applies to complementary, patient-reported experience measures (PREMs) which aim to capture elements such as route of administration of treatment, impact on caregivers and burden of disease. A recent literature review on use PREMs in 12 HTA bodies uncovered that only $11 \%$ of HTA reports published after 2012 considered these measures to some extent [16].

\section{Incorporating patient preferences in assessments of health technologies}

This probably is the most contentious area of greater patient involvement. In Europe there are two main processes for making decisions about the adoption of new health technologies [17]. The first approach, used in France and Germany, uses assessments of 'added clinical value' based on an assessment of the clinical evidence for the technology concerned. It is not clear whose preferences are most influential in this approach and whether, when making assessments of added clinical value, clinicians mirror the preferences of patients. However, it may be possible for those making the decisions to be made aware the views of patients on the trade-offs between the various clinical endpoints being considered.

The other approach, used in a number of Northern European countries, is to undertake assessments of the incremental cost-per quality-adjusted life-year (QALY) gained for new health technologies and to compare the ICER with a decision-making threshold, representing either the maximum willingness-to-pay for a given health gain, or the opportunity cost of the health displaced by discontinuing other treatments to make room (in the budget) to accommodate the new technology. In so far as preferences are used in this approach, they are normally the population-based health state preference values used in health-related quality-of-life instruments such as the EQ-5D. As in the clinical approach discussed above, it may be possible for those making the decisions to be made aware of the views of patients. This is already the case in some jurisdictions, through the presentation of patients' experiences to the committee making the recommendations on the use of the treatment or technology. It may be also possible to use health state preference values based on the preferences of patients, but these are not often used to estimate the QALYs gained.

Some of the preference measurement approaches, such as discrete choice experiments (DCEs) naturally lend themselves to more consideration of these additional dimensions than the standard approaches for estimating QALYs. However, their use in HTAs for decision-making has so far been limited [18]. The reasons for this are not clear, although the standardization offered by QALYs is attractive to those decision-makers having to make a sequence of decisions, such as determining which treatments should be included on a 'positive list', or in the health insurance package. However, by their very nature, DCEs are often bespoke, whereas 
decision-makers often value standardization in their decision-making procedures. Therefore, using these measures might be a challenge for those performing and using HTAs [19].

However, it may be possible to standardize the attributes and levels used in DCEs in the healthcare field. It would also be possible to conduct DCEs with a representative sample of the general public, as opposed to patients. A European Union initiative, IMI-PREFER, seeks to establish recommendations to support guidelines for industry, regulatory authorities and HTA bodies on how and when to include patient perspectives on benefits and risks of medicinal products. However, out of 31 partners in the consortium, only one is an HTA body (www.imi-prefer.eu).

\section{Other approaches: recognizing patient heterogeneity by offering more choice}

Although the precise approach to decision-making differs across jurisdictions, the aim of HTA is to recommend a preferred course of action. Often this is a recommendation about the use of a particular health technology, but sometimes involves recommending a strategy for prevention, diagnosis or treatment. The definition of 'preferred' is sometimes unclear, but often means the strategy that is most clinical and cost-effective. One way of being more patient centric would be to recognize that the preferred strategy might not be the same for all patients, because of heterogeneity in clinical characteristics or patient preferences. This would suggest that a choice of treatments could be offered, rather than a single 'preferred' treatment.

In England, a jurisdiction where cost-effectiveness plays a central role in HTA recommendations, there are some examples where choice has been offered at additional cost, but within the threshold of 'acceptable' cost-effectiveness. For example, in a NICE clinical guideline on caesarian section it was recommend that women could request a planned caesarian section, rather than a vaginal birth, if after counseling they still had anxieties about childbirth [20]. NICE also recommended that, instead of basal insulin therapy, patients with type 1 diabetes could be offered more expensive longacting or quick-acting insulin therapy if twice-daily basal insulin therapy was not acceptable based on patient preferences [21].

There may be other examples of offering choice, in situations where although there was an increase in cost, offering choice including the more expensive therapy was still within the acceptable range of cost-effectiveness. Another situation could be one where, to offer equal access to care for all patients, much greater costs would be incurred. For example, patients living in remote areas may need expensive transport to receive care, or may require other special provisions, such as mobile screening services. Even more controversially, treatments for patients with rare conditions may be more expensive to treat, so offering equal opportunity for care to these patients would have a substantial budgetary impact and consequent restrictions of care to other patients if the budget is fixed [22]. Clearly, there are a number of considerations in these cases, but there is a growing literature on how efficiency-equity choices could be addressed in conducting economic evaluations as part of HTA [23].

\section{Conclusions}

Should HTA be more patient centric? The main driving force behind HTA should remain the preferences of the whole population served, whether this be the enrollees in a health plan, or the individuals having entitlement under national health insurance or health service. In these situations the provision of health care is largely funded collectively, although there are often some patient payments. Under conditions of collective funding it would be wrong to have health care provision driven by individual patients' preferences, since this might run counter to collective wishes. Therefore, on occasions difficult choices are required to reconcile competing interests. In this editorial we argue that even within these constraints there are several initiatives than can be taken to make HTA more patient centric. This can be done by encouraging patient engagement in HTA process, expanding the HTA methods to include patient-reported evidence, and other approaches, such as offering more choice, within the bounds of cost-effectiveness or based on a formal consideration of efficiency-equity trade-offs.

\section{References}

1. Perfetto, E.M.: ISPOR's initiative on US value frameworks: a missed opportunity for ISPOR and patients. Value Health 21(2), 169-170 (2018)

2. Westrich, K.: Current landscape: value assessment frameworks. Washington, National Pharmaceutical Council, 2016 (www.npcow .org)

3. Slejko, J., Mattingly, T.J., Mullins, C.D., Perfetto, E., dosReis, S.: Future of patients in healthcare evaluation: the patient-informed reference case. Value Health 22(5), 545-548 (2019)

4. Sanders, G.D., Neumann, P.J., Basu, A., et al.: Recommendations for conduct, methodological practices, and reporting of cost-effectiveness analyses: second panel on cost-effectiveness in health and medicine. JAMA 316, 1093-1103 (2016)

5. Suarez-Almazor, M.E., Conner-Spady, B.: Rating of arthritis states bypatients, physicians, and the general public. Implications for cost-utility analysis. J Rheumatol 28, 648-656 (2001)

6. Goodwin, E., Green, C., Hawton, A.: What difference does it make? A comparison of health state preferences elicited from the general public, and from people with multiple sclerosis. Value Health (2019). https://doi.org/10.1016/j.val2019.08.009

7. Ogorevc, M., Murovec, N., Bolanos Fernabdez, N., Prevlnik, Rupel V.: Questioning the differences between general public vs. 
patient based preferences towards ED-5D-5L defined hypothetical health states. Health Policy (2017). https://doi.org/10.1016/j.helat hpol.2017.03.011

8. Torbica, A., Tarricone, R., Drummond, M.F.: Does the approach to economic evaluation in health care depend on culture, values, and institutional context? Eur J Health Econom 19(6), 769-774 (2018)

9. Drummond, M., Tarricone, R., Torbica, A.: Assessing the added value of health technologies: reconciling different perspectives. Value Health 16(1), S7-S13 (2013)

10. National Institute for Health and Care Excellence. Citizens Council. www.nice.org.uk. Accessed Feb 202020

11. Bijlmakers, L., Jansen, M., Boer, B., et al.: Increasing the legitimacy of tough choices in healthcare reimbursement: approach and results of a citizen forum in the Netherlands. Value Health 23, 32-38 (2020)

12. Perfetto, E.M., Harris, J., Mullins, C.D., dosReis, S.: Emerging good practices for transforming value assessment: patients' voices, patients' values. Value Health 21(4), 386-393 (2018)

13. Garrison, L., Neumann, P., Willke, R., Basu, A., Danzon, P., Doshi, J., Drummond, M.F., et al.: A health economics approach to US value assessment frameworks-summary and recommendations of the ISPOR special task force report [7]. Value Health 21, 161-165 (2018)

14. Lakdawalla, D.N., Doshi, J.A., Garrison, L.P., Phelps, C.E., Basu, A., Danson, P.M.: Defining elements of value in health care-A health economics approach: An ISPOR special task force report [3]. Value Health 21, 131-139 (2018)

15. Ciani, O., Federici, C.: Value lies in the eye of the patients: the why, what and how of patient-reported outcomes measures. Clin. Ther. 42(1), 25-33 (2020)
16. Sarri, G., Kenny, J., Freitag, A., et al.: How frequently is patient experience formally assessed in health technology assessments? Results from a systematic literature review. Value Health 21(Suppl 3), S204 (2018)

17. Drummond, M.F., de Pouvourville, G., Jones, E., Haig, J., Saba, G., Cawston, H.: A comparative analysis of two contrasting European approaches for rewarding the value added by drugs for cancer: England versus France. PharmacoEconomics 32(5), 509-520 (2014). https://doi.org/10.1007/s40273-014-0144-z

18. Marsh, K., van Til J., Molsen-David E et al.: Health preference research in Europe: a review of its use in marketing authorization, reimbursement and pricing decisions. Value Health 2020 (in press)

19. Mott, D.J.: Incorporating quantitative patient preference data into healthcare decision making processes: is HTA falling behind? Patient (2018). https://doi.org/10.1007/s40271-018-0305-.9

20. National institute for Health and Care Excellence November: CG 32 Caesarian Section. NICE, London (2011)

21. National institute for Health and Care Excellence August: NG 17 Type 1 Diabetes in Adults. NICE, London (2015)

22. Drummond, M.F., Towse, A.: Orphan drugs policies: a suitable case for treatment. Eur J Health Econom 15(4), 335-340 (2014)

23. Cookson, R., Mirelman, A.J., Griffin, S., et al.: Using cost-effectiveness analysis to address health equity concerns. Value Health 20(2), 206-212 (2017)

Publisher's Note Springer Nature remains neutral with regard to jurisdictional claims in published maps and institutional affiliations. 